\title{
Le trasgressioni dell'uomo bizantino. Alternative ai costumi sessuali all'interno ed al di fuori del vincolo matrimoniale: eunuchi ed immaginario sessuale
}

Antonio Pio Di Cosmo

Universidad de Córdoba, España apiocosmo @outlook.it

Recibido: 12/6/2018. Aceptado: 23/11/2018.

\section{Riassunto}

Questo contributo analizza un metodo cognitivo per ricondurre a sistema la presenza degli eunuchi e discerne dello sviluppo dell'idea della virilità nella cultura bizantina. Si vogliono annoverare i problemi-base concernenti la rappresentazione della mascolinità, quali codici cognitivi utili a revisionare una certa idea dell'uomo 'completo' rispetto ai castrati. Questa ricerca applica le conoscenze in materia antropologica correlate alle categorie epistemiche della sociologia e le riconduce al lavoro di sistematizzazione della dottrina; si concludono così le questioni circa i problemi di rappresentazione dell'homus byzantinus e della femina byzantina rispetto all'eunuco. In questo modo, si approfondiscono le diversità di genere avverso la capacità sessuale degli eunuchi, che modellano la concreta percezione di un tertium genus.

The transgressions of the Byzantine man. Alternatives to sexual costumes inside and outside the conjugal constraint: eunuchs and sexual imaginary

\footnotetext{
Abstract

This contribution analyzes a cognitive methodology and takes to the system the presence of the eunuchs, also focuses the development of manhood's idea in the Byzantine culture. It recognizes basic problems concerning the representation of manhood, as cognitive codes, that revises the idea of the 'complete' man, compared to the neutered man. This research uses the anthropological knowledges referred to the epistemic categories of sociology and puts those in relation to the work of doctrine. Therefore, it examines the problems concerning the representation of homus byzantinus and femina
}

\section{Parole chiave}

eunuchi virilità casti viri endiadi uomo/donna tabù
Keywords

Eunuchs manhood casti viri

hendiadys men/woman taboo 
byzantina adverse the eunuchs. In this way, it scrutinizes the differences of genders adverse the sexual capacity of the eunuchs, which models concrete perception of a tertium genus.

\section{Introduzione}

La presente ricerca è volta ad indagare gli spazi d'azione riservati agli eunuchi avverso il normale menage concernente la vita sessuale dell'uomo bizantino dei secc. IX-XI. Nonostante si abbia a considerare un preciso arco cronologico di riferimento, appare altrettanto opportuno approfondire il fenomeno sul piano sincronico e diacronico. In tal modo si evidenzia la continuità di determinati atteggiamenti verso la categoria anche nel lungo periodo, perché essi non sono null'atro che sottoprodotti di una delle strutture con cui l'uomo bizantino percepisce il mondo e sistematizza una situazione eccezionale come l'eunuchia, almeno rispetto all'endiadi uomo-donna. Una diversità di condizione, che implica anche differenti possibilità di vivere la sessualità, sempre entro i limiti del tipo di menomazione a cui il singolo eunuco è stato sottoposto. Condizione che può suscitare fantasie nel maschio in particolare ed offrire occasioni di trasgressione a quest'ultimo. Ciò reclama una revisione della percezione della realtà quotidiana della società bizantina e delle preferenze sessuali dei suoi membri, almeno rispetto all'immagine aulica che le strategie di auto-rappresentazione della stessa offrono.

\section{Eunuchi e virilità: problemi di autorappresentazione dei generi}

È ben noto l'orrore che prova l'uomo bizantino avverso tutto ciò che lo può far sembrare effeminato, l'oltraggio più temuto è quello che mette in discussione la sua virilità. Un timore a maggior ragione giustificato, se si pensa al locus caustico che si adopera per identificare i graeci: 'effeminati' (Pontieri, 1925-1928:10, I, 1, 1; Ducellier, 1980:21; Guillard, 1943:196-238; Hadley, 1998). Questo timore spiega pure una condotta crudele: l'evirazione post mortem, quale massima forma di vilipendio, come quella accorsa alla salma del cesare Bardas (865 d.C.), dopo il suo assassinio da parte di Michele III (840-867 d.C.). Un timore che rimanda ad un conflitto psicologico vero e proprio, vissuto dall'uomo 'completo', rispetto alla condizione di eunuco, quale 'diverso' per antonomasia. Una posizione che origina dall'idea fatta propria dall'inconscio collettivo circa le modalità con cui il maschio deve vivere l'esperienza sessuale, avverso gli ovvi limiti a cui sono sottoposti gli eunuchi. Una condizione di fatto che genera una situazione di marginalità per questi ultimi, dovuta, in fin dei conti, ad una causa insanabile. Una marginalità che non è nulla più di un sottoprodotto della cultura in cui matura una tale idea di mascolinità, quale 'dramma' che non conosce 'catarsi' ed investe coloro che non possono vivere appieno questa componente della vita (Ducellier, 1980:21; Corsi, 1991:261; Tougher, 1997:168-184). Una situazione che viene accentuata dalla legge, la quale ha l'attenzione di distinguere la condizione d'eunuco in ragione dell'origine di

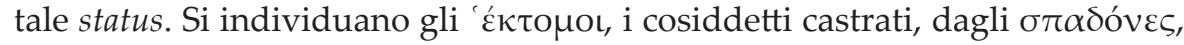
che sono incapaci di procreare per difetto genetico o malattia.

La presenza degli eunuchi nel menage quotidiano, pubblico e privato, da origine ad un acceso dibattito che concerne la percezione di un eventuale 
genere a sé (Neil; Garland, 2016). Nonostante la presenza degli eunuchi nella società bizantina fosse ben radicata e addirittura i loro servizi altamente richiesti negli ambienti medio-alti, non mancano dei pregiudizi verso questa categoria (James, 1997; James, 2009). Pregiudizi che non sono nulla più che cause ed, al contempo, sottoprodotti di una certa teoria dei generi presente in nuce alla cultura bizantina. Se Kazdan (1982) ha percepito come autonome le categorie dell'homo byzantinus e della femina byzantina, ben più difficile è classificare gli eunuchi, sia rispetto al genere che circa l'appartenenza a Bisanzio. Costoro fino alla prima fase della Media Bisanzio sono in genere stranieri. Giustiniano con la forza di legge ha vietato la creazione di eunuchi all'interno dell'Impero; un divieto che si fa assoluto per i cittadini romani (Ravegnani, 1989:32-34; Hopkins, 1963:62-80). Solo con la Casa macedone le leggi giustinianee sulla castrazione entro i confini della Basileia si attenuano e si fa largo alla possibilità di 'eunichizzare' lecitamente anche i romei. Non meraviglia che alcuni membri delle più importanti famiglie, per acquisire $o$ confermare il potere esercitato a corte, scelgano questa via per uno dei loro figli (Herrin, 2003:214-215). Le leggi rendono più 'accessibile' una pratica odiosa, fin a quel momento ammessa eccezionalmente e riservata solo agli imperatori deposti ed alla loro discendenza, al fine di eliminare ogni possibile pretendente al trono.

Tuttavia è la capacità sessuale degli eunuchi che rende più complessa la riduzione a sistema di una condizione sui generis rispetto all'endiadi uomodonna. Una capacità che apre a pratiche alternative puntualmente censurate dalla Chiesa.

Una sistematizzazione che richiede una rivisitazione delle categorie della cultura bizantina, al fine di meglio comprendere la percezione che l'uomo comune ha della condizione di eunuco. Le loro attitudini, allora, si devono riflettere necessariamente in stereotipi maschili e femminili propri della cultura bizantina. È difatti impossibile violare le strutture mentali. Per comprendere l'anomalia si è costretti a muoversi al loro interno, forzandole progressivamente, al fine di aggiornarle. Un buon eunuco sarà allora mascolino, come lo sono già state le ottime donne, che vengono additate come 'femine virili'; il cattivo eunuco al contrario risponderà a qualità tutte femminili. Siamo di fronte ad una stigmatizzazione di genere endogena al contesto sociale di riferimento. Una soluzione che costituisce una sorta di esperimento, i cui medi cognitivi sviluppano e si giustificano nelle categorie culturali di chi pone in essere l'operazione.

\section{La Chiesa e gli eunuchi. Riflessioni a margine di un rapporto ambiguo}

Sant'Andrea il folle (IX sec.-936 d.C.) col suo ammonimento all'eunuco offre un vivido spaccato delle pratiche sessuali della precoce Media Bisanzio, interpretate entro i limiti della visione dei ranghi ecclesiastici che commissionano lo scritto agiografico. Ranghi che da sempre disciplinano in maniera minuziosa la vita sessuale della società e censurano ogni eccesso di libido. Alla mannaia ecclesiastica non possono sfuggire gli eunuchi, che ovviamente posseggono una loro libido e la esercitano nei limiti della propria condizione fisica. La feroce censura del santo fa percepire quanto queste condotte fossero comuni al tempo in cui si pone mano al testo, tanto che la Chiesa deve intervenire con aggressività per reprimerle. 
1. Thom. Aquin., Sum. Theo., II, III, q. 154 a 11 co.
Eppure l'agiografo si limita ad evocare un locus 'classico'. E se definisce questo 'colofone' (inteso come 'colui che abusa del colon'), non aggiunge nulla alla panoplia di vizi che tradizionalmente si attribuiscono a questa categoria. Opera una reificazione della persona, a causa di quello che è considerato un vizio. Ricorre infine ad un repertorio di genere, che si ritrova tutto nell'apostrofe invettiva che questi pronuncia.

Repertorio condiviso dalla linea di pensiero che in Occidente genera sul più lungo periodo quei manuali ad uso dei curati, che sono definiti Confessionali. Ciò dimostra che queste elaborazioni sono dei sottoprodotti della medesima struttura e sono pertanto destinati a durare allungo.

Il santo, ponendosi su questa linea comune, può giovarsi di quelle elaborazioni circa il Foro interno. Prima inquisisce il peccato, denudandone gli aspetti psicologici, fin tanto da evidenziarne la mania, perché determinate pratiche sono considerate insane tout court, in quanto ritenute contro natura in sé. Suscita poi la vergogna nel peccatore con opportuni epiteti, seguendo un codice che è anch'esso 'classico' nell'omiletica e perdura nel lungo periodo. Segue l'ammonimento dell'inferno con le sue fiamme, che è il più abusato dei loci di genere, dacché compare in ogni racconto agiografico e per ogni tipologia di peccato a vantaggio di una rapida conversione del peccatore.

Si riscontra un immaginario di genere, tutto fatto di formule che non sono niente affatto che una novità. Infine si perora una pia credenza: la sodomia è una pratica solo degli uomini e non degli altri esseri viventi. Un'idea diffusa nel panorama teologico nel più lungo periodo, che si ritrova anche in San Tommaso d'Aquino. ${ }^{1}$ Entrambi gli ecclesiastici allora sembrano rifarsi ai trattati di biologia animale che sviluppano da presupposti risultanti, con loro buona pace, errati.

Anche il locus della vergogna innanzi all'angelo è altrettanto tipico e comune all'Occidente e si può ritrovare ben oltre la cronologia di riferimento a dimostrazione della longevità di queste elaborazioni. Circa la sodomia S. Caterina da Siena, dopo un colloquio col Cristo, potrà dire che non solo gli angeli si vergognano nell'assistere a certe pratiche, ma lo fanno persino i demoni. Perché la loro natura è pur sempre angelica, indi pura, ed il veder compiere atti che puri non sono gli causa disagio. ${ }^{2}$ Siamo di fronte ad un espediente che costituisce un'altra elaborazione comune, riconducibile anch'esso a sottoprodotto di un'unica struttura; ciò a deroga di quella tolleranza verso un fenomeno molto diffuso in Oriente.

L'ammonimento del santo è dunque funzionale ad arginare proprio quella proliferazione di pratiche sessuali che violano il fine esclusivo a cui la Chiesa indirizza la copula carnalis: la procreazione. Queste pratiche già cadono sotto la mannaia del veto biblico contenuto nel Levitico (Lev. 18.22; 20.13) e ribadito nell' Apocalisse, laddove gli impuri sono esclusi dalla Gerusalemme del cielo (Ap. 21.8). È noto che la religione, specie quella orientale, tende a circoscrivere tali pratiche: un tabù che si ritrova già nel Libro egiziano dei morti. La Chiesa dunque non si inventa nulla.

Nonostante il veto religioso anche a Bisanzio queste pratiche vengono esercitate nel segreto delle camere da letto e gli ammonimenti morali ed il prospetto di pene eterne, maturate nell'humus culturale del Medio Oriente, non sembrano sufficienti ad arginare il fenomeno. 
Allorché Epifanio, il compagno del santo, prova a intessere una difesa ricordando che certe pratiche sviluppano a corollario della condizione di servo, propria dell'eunuco medio, non fa altro che perorare una posizione indifendibile (Ringrose, 2007). Anzi scade nella defensio peccatorum. La prospettazione delle pene fisiche che aspettano il servo-eunuco, che contraddice la volontà del padrone, non sono sufficienti a giustificare la sua passività avverso i desideri peccaminosi. È davvero una causa persa. La clausola di excusatio: coactus tamen non voluit, quale clausola generale, non sembra operare affatto nel caso in osservazione. La condizione di servo non giustifica l'accondiscendenza alle 'opere del diavolo', che si traducono in 'un'anomalia maledetta e disgustosa che non compiono nemmeno gli animali'; il servo si deve limitare a soddisfare le necessità del padrone (Rydén, 1995:1119-1290; 24).

L'agiografo ha a sancire l'inesistenza di ogni excusatio. La sodomia va censurata sempre e comunque. La sentenza del santo stigmatizza la natura oggettiva della condotta al di là di ogni elemento volitivo.

La Chiesa, che dispiega la sua autorità nel Foro interno, solitamente tende a fornire tutta una serie di attenuanti, che emergono indagando fin nelle pieghe più profonde dell'anima. Un interesse per la fase volitiva che viene escluso nel caso in esame. Si raffronta davvero una causa persa! Non solo dal punto di vista retorico, ma anche sostanziale, quale situazione eccezionale avverso i metodi ordinari d'inquisizione. La pratica sessuale non finalizzata alla procreazione non ammette né deroghe, né attenuanti, nemmeno sul piano psicologico; né il timore di un male più grande giova ad assolvere il reo della condotta. Andrea sentenzia l'unica possibilità contro tale abuso da parte dei padroni: la resistenza!

L'agiografo reinterpreta l'Evangelo di Matteo e lo pone alla base del suo assunto. Il rifiuto di tali pratiche è considerato opera secondo giustizia e l'eventuale castigo viene subito assimilato al martirio. Resta nulla più che la resistenza, perché la castità propria e la censura della libido altrui sono le uniche vie per preservare la virtù.

L'apostrofe del santo viene poi presentata come atto straordinario: la condotta merita che questi intervenga in prima persona dimostrando tutto il suo dissenso. Siamo di fronte alla falsificazione di un codice: l'episodio si pone nella duplice funzione di admonitio peccatorum e di narrazione edificatoria delle virtù del santo, che legge nelle anime e mostra la sua potenza taumaturgica. L'intransigente agiografo ha come scopo la repressione totale della sodomia e, perciò, colpisce un corollario della condizione di eunuco.

Dal punto di vista sociologico l'aneddoto offre uno spaccato ancor più interessante. Gli attori di questo dialogo sono entrambi oggetto di pregiudizio. Il santo, quale 'folle per Dio', rappresenta una nuova strada per la santità, perché si offre da sé all'umiliazione ed in ragione della sua peculiare vocazione, si pone volontariamente in situazioni ambigue. Osserva la vita da un punto di vista privilegiato, almeno per quel che riguarda le interazioni sociali. Vive la strada e quanto offre di alternativo alla mentalità aristocratica, quella che connota la produzione letteraria bizantina (Mango, 1982:97-313; Patlagean, 1992:27-37). Una situazione eccezionale rispetto alla casistica agiografica del monaco, quale modello privilegiato di santità in voga dal sec. VIII in poi, che vive la sua virtù entro le mura del monastero. Pertanto il 'folle per Dio', a causa del suo quotidiano, diviene la figura più qualificata ad esprimere un punto di vista che, paradossalmente, è quello della gerarchia. 
3. Typikon dell'imperatore Giovanni Tzimisce, cit. in Thomas; Constantinides Hero (2000:ก.12).

\section{Raccontare gli eunuchi. La percezione sociale fra voluttà e castità: esempi di indicatori letterari}

L'eunuco sembra davvero suscitare fascino e turbamento in colui con cui si raffronta. Un turbamento di tipo sessuale, come dimostra l'esistenza di descrizioni voluttuose riscontrabili nelle fonti. Una voluttà che, alla luce di una mentalità permeata dalla morale cristiana, viene percepita quale fonte di disagio. E se l'attrazione per questa categoria è vissuta come esperienza peccaminosa, essa ha il suo contraltare nel gusto per il proibito e nel fascino della trasgressione. E la Chiesa sa bene questo.

Un pregiudizio che deve essere davvero fondato, dato che si teme non solo per l'emotività dell'uomo bizantino medio, che intrattiene un normale menage familiare, ma si ha persino a dubitare dell'onestà morale dei 'santi' monaci.

Non a caso la regola firmata da Giovanni Tzimisce per l'Athos impone: "ti ordino di non accogliere uomini senza barba ed eunuchi che vengono alla Montagna per ricevere tonsura (...). Inoltre se qualche abate o kelliotes non rispetta le mie norme ed introduce nei suoi domini o nella sua cella un eunuco od un ragazzo (...) crediamo sia la cosa miglior espellerlo dalla Montagna". ${ }^{3}$

Siamo di fronte ad una prova provata, perché l'ambiguità dell'eunuco può sempre tentare il maschio bizantino, anche se monaco. Molti monasteri infatti sono restii ad accettare eunuchi ed anche fanciulli al fine di evitare il sorgere di fantasie da parte dei più anziani, ma ancor più l'instaurarsi di situazioni ambigue. Dopo tutto anche quello della sodomia è un altro locus tipico delle dispute interne alla Chiesa d'Occidente. Un locus di cui spesso si abusa, portandolo ai limiti del parossismo. Un abuso come l'accusa di sodomia verso il centenne S. Romualdo ai danni di un giovane discepolo (Cantarella, 2004:128129). A ragione si può affermarne l'infondatezza, dato che il santo, seppur ne avesse avuto la volontà, non avrebbe avuto le forze per attuare l'intento. Perciò a riguardo nulla quaestio. Tuttavia, se i detrattori hanno a ricorrere ad una situazione così paradossale, il fenomeno non deve essere del tutto sporadico. Un dato di fatto che crea un locus polemico, utilizzabile secondo evenienza. Anche quest'espediente può essere considerato un'elaborazione riconducibile alla medesima sovrastruttura comune.

Il timore concernente l'insorgere di turbamenti negli integerrimi membri delle comunità maschili non deve essere considerato un elemento ostativo in assoluto all'accesso degli eunuchi alla condizione di religioso. Costituisce piuttosto una 'clausola generale di garanzia', limita a determinate situazioni.

Il problema non si pone affatto per le monache. Anzi il clero eunuco è quello preferito per l'assistenza spirituale dei monasteri femminili e la celebrazione delle liturgie all'interno di questi. L'asessualità degli eunuchi, al contrario, è una precisa garanzia per il loro corretto ministero, tanto che molti statuti di fondazioni prevedono questa 'clausola di salvaguardia' per le monache, al fine di evitare la tentazione dovuta alla presenza di un uomo tra loro.

Per meglio percepire il turbamento di natura sessuale che viene suscitato da questa categoria, basta riferirsi alla voluttuosa descrizione che emerge nella stessa vita di S. Andrea. L'eunuco si presenta come soggetto ambiguo, che mostra alcuni attributi del sesso femminile, ma come una donna (o forse più) deve suscitare libido, proprio perché non è donna. S'offre al lettore un ritratto 
sensuale del genere: "Il suo volto era come una rosa, la pelle del suo corpo bianca come la neve, era ben modellato, biondo, dotato di una morbidezza insolita, e profumava di muschio da lontano" (Rydén, 1995:1119-1290; 24).

E se tale suggestione condiziona l'agiografo, che dopotutto si volge all'edificazione delle anime, si può solo immaginare quali sensazioni gli eunuchi possano suscitare nell'uomo comune e soprattutto nei soggetti già inclini a scelte alternative. Si rende così più tangibile il pericolo intuito dalla Chiesa, che rifugge pratiche sessuali alternative, che si contrappongono alla procreazione.

Una voluttà che ha diretti paragoni in letteratura. Altrettanto ambigua risulta la descrizione degli eunuchi che perviene da un'altra fonte più tarda, l'epico romanzo di Digenis Akritas. Costui riceve in dono di matrimonio dallo zio della sposa dieci eunuchi adolescenti, che vengono descritti in modo altrettanto sensuale: "senza sesso e di bell'aspetto, con adorabili capelli lunghi, / vestiti alla foggia persiana con abiti di seta, / con belle sciarpe dorate attorno ai loro colli" (Odorico, 1995, cit. in Herrin, 2008:216).

Il narratore non si limita a suggerire la forma corporis degli eunuchi, ma calca la mano sull'elemento esotico, con particolare attenzione all'outfit ed al suo dettaglio prezioso. Siamo di fronte ad un altro codice, che ha parecchie implicazioni e rivela molto sul versante psicologico di chi compone; una percezione che deve essere necessariamente condivisa dai lettori, ma anche dall'uomo bizantino nel più lungo periodo. Si raffronta una tecnica di somministrazione dei significanti, che sfrutta alcuni loci-base per descrivere la categoria. E se il richiamo al locus della bellezza delicata e femminea da solo deve incuriosire, si ha piuttosto a colpire l'auditorio con un'opportuna precisazione: l'indeterminatezza del sesso. Si concentra così l'attenzione sugli attributi che devono suscitare la libido nel lettore, dettagli come i capelli lunghi, attributo di seduzione femminile. Le donne bizantine e quelle del mondo mediterraneo sono solite mostrarli nell'intimità della casa, mentre in pubblico li portano ben nascosti entro le cuffie. Un dettaglio che l'iconografia registra puntualmente per le sante. Un costume quindi condiviso da tutte le donne di buona famiglia. Le uniche che mostrano i capelli invece sono le ballerine, come quelle rappresentate in una miniatura del codice 479 della Cinegetica dello Pseudo-Oppiano del sec. XI, ora alla Marciana di Venezia, e Maria Maddalena che, non a caso, è tradizionalmente identificata con una prostituta. L'evocazione dei capelli da sola può suscitare lascivia. I capelli lunghi sappiamo poi che sono attributo dei bambini fin all'inizio dell'adolescenza; una precisazione non senza implicazioni. Cosa che allude a quelle che possono essere alcune preferenze sessuali dell'uomo medio bizantino. Opzioni che includono un'altra trasgressione dell'immaginario sessuale: quella piaga che è la pederastia.

Al dettaglio che accende le fantasie si aggiunge la veste esotica e il fasto della sciarpa d'oro, che assimilano costoro ad abitanti del Paese di cuccagna. Una cuccagna che non può essere esente da sottili allusioni sessuali.

Eppure la loro bellezza e sensualità non può essere ridotta ad una sola visione negativa. Questa condizione può essere pure presentata in una luce positiva, allorché legata al Sacro Palazzo ed alle sue funzioni. È la santità istituzionale della 'divinità imperiale' che ne purifica parzialmente l'impatto. Tanto che l'Oneirocriticon arriva addirittura a paragonarli agli angeli (Cosentino, 2006:573607; Carile, 2003:589-656; Carile, 2003:27-32). Da tentatori, questi 'casti viri', assumono così uno status che è il loro diretto opposto. Gli eunuchi, che hanno perso il sesso, si possono assimilare agli incorporei. Un espediente utilissimo 
dal punto di vista della retorica del potere, perché permette al basileus di autorappresentarsi nelle sue epifanie davvero come il "Signore tra i suoi santi" (Zc $14,5)$. Siamo di fronte ad un abile gioco di rimandi metaforici, che agevola lo scivolamento di significanti e l'arricchirsi del nucleo originario di nuove accezioni, in origine non previste, ma accettate di buon grado dalla collettività. Un altro tentativo di messa in codice utile a sistematizzare quella realtà eccezionale rispetto all'endiadi uomo-donna.

Un codice che infine redime la categoria dai soli appetiti sessuali. Sicché l'assenza del sesso non viene costretta al 'dramma' di una condizione di marginalità in termini assoluti. Come ogni codice anche questo deve avere il suo contrappeso ideologico, come accade ad ogni espressione materiale del potere imperiale. La folla degli eunuchi viene così apostrofata dall'imperatore Costantino VII con sarcastica invettiva, poiché il loro numero a Palazzo è paragonabile a quello delle mosche in un ovile d'estate (Bekker, 1838:318, n. 8-10). E se la riabilitazione della figura dell'eunuco implica necessariamente il sottoporsi a quel gioco di mistificazione e sublimazione della realtà che il protocollo mette in scena, la stessa operazione trova un suo calmiere in quel sentore per l'effimero, che la mentalità bizantina rifugge. Eppure la frivola corte macedone, a mezzo di un meccanismo di pesi e contrappesi ideologici, almeno formalmente allontana da sé lo spettro della vanità, implicito nella lode magnificatoria celebrata attraverso il protocollo. La nobilitazione della condizione dell'eunuco è dunque uno dei tanti tentativi con cui il basileus, come sottolineato efficacemente dallo stesso Costantino VII, tenta di mettere in atto, per quanto è possibile ad un uomo, la Christomimesis (Dagron, 2007:210). Questo gioco, tutto interno alla corte e fatto di stratagemmi volti ad enfatizzare alcuni aspetti con cui la monarchia romano orientale si racconta, contribuisce alla ridefinizione della condizione degli eunuchi.

Il tentativo spiega come un monaco eunuco, Cosma di Pafagonia, in visita alla tomba di S. Luca a Steiris, possa diventare il destinatario di una visione. Lo si invita a restare presso la tomba del santo al fine di renderla più bella e decorosa. Un aneddoto che si inserisce in un più ampio disegno volto alla riabilitazione morale della categoria, perché, in fin dei conti, il raggiungimento della santità di uno dei suoi esponenti funge da prova regina. L'adynaton narrato dall'agiografia completa la metafora angelica della teologia del potere e rende fatto compiuto la redenzione dello stato d'eunuco. Come per l'uomo 'completo', anche per quest'ultimo il marchio della santità consta nella continenza e nella scelta di una vita devota (Herrin, 2003:216).

\section{Eunuchi, norme e pratiche sessuali. Questioni sulla rappresentazione della virilità}

Come noto, in tutti i sistemi imperiali molti uomini per vivere una vita dignitosa scelgono l'auto-castrazione divenendo servi 'sicuri'. Ciò allorquando la condizione non gli viene imposta per traversie della vita o dalla stessa famiglia, che vuole migliorare il proprio status. Si può allora parlare dell'eunuchia come fenomeno 'globale' e trasversale alle culture, che si spalma nel più lungo periodo.

La comprensione dell'atteggiamento verso gli eunuchi e le loro pratiche sessuali, che possono incitare il maschio alla trasgressione, obbliga sul piano diacronico a considerare la comune percezione delle condotte sessuali alternative. Pratiche che stimolano in particolare la produzione legislativa di 
Giustiniano. Quest'imperatore interviene su un fenomeno più antico e che gli sopravvivrà: vieta la castrazione all'interno dell'Impero innanzitutto, almeno dei cittadini liberi. Il divieto non vale però per i non romani ed i prigionieri di guerra. La gran parte degli eunuchi conseguentemente viene 'creata' al di fuori dei confini imperiali. Stando a quanto racconta Procopio di Cesarea (490-565 d.C.) è possibile acquistarne in Abasgia. ${ }^{4}$ Più specificatamente, per quel che riguarda la nostra cronologia di riferimento, è Liutprando (920?- 972 d.C.) a fornirci informazioni. Questi afferma che un ruolo importante nella diffusione di queste risorse umane lo gioca la Spagna ${ }^{5}$ e la città di Pechina, ove gli ebrei ne gestiscono la produzione come Ibn Hawqal (978 d.C.) ha ad attestare. Quest'ultimo poi qualifica l'eunuchia come fenomeno tutto bizantino, ignorandone l'origine più antica (Kramers, 1964:vol. I, 198-199). In quella città i mercanti di Verdun possono ricavare grossi guadagni da tale commercio, nonostante le invettive papali che mirano a contenere il fenomeno ed a salvare dalla castrazione almeno i cristiani.

L'esistenza dei divieti normativi non esclude che si possa essere eunuchizzati anche entro il territorio della Basileia. All'interno dell'Impero tale pratica sopravvive formalmente con funzione punitiva avverso gli oppositori politici.

Tuttavia la legge non si limita solo ad intervenire sulle condizioni che favoriscono l'eunuchizzazione, ma si prefigge di disciplinare la vita sessuale del bizantino, che oltre alla moglie ed a donne che esercitano il sesso di mestiere può ricorre ad alternative. Della ricerca di questo tipo di favori un buon indizio viene da Procopio. Questi nelle Storie segrete riferisce che Teodora (497-548 d.C.) sin da bambina è costretta alla prostituzione, e sebbene non ancora matura per un tale tipo di azione, si offre a rapporti assimilabili alla pederastia maschile. ${ }^{6}$ Pratiche che rientrano nelle possibilità concesse agli eunuchi di vivere la propria sessualità.

Un fenomeno forse fin troppo diffuso, se si considera che si deve intervenire con una normativa volta alla repressione puntuale (Dalla, 1987). Per far ciò si ricorre ad uno strumento erga omnes come l'editto, diretto ai cittadini di Costantinopoli. La "libidine cum masculis" [libidine con l'uomo], che Giustiniano moralisticamente definisce "infandam" [infame], esige le attenzioni imperiali (Just., Inst. 4, 18, 4). Questa, sin dalla Nov. 77 del 535, viene inserita come fattispecie ex novo a corollario della figura criminis della bestemmia; l'azione è ricondotta a quell'alea in quanto atto contro natura e contro Dio.

La Nov. 104 del 559, l'Edictum ad costantinopolitanos de luxuriantibus contra naturam, precisa la figura criminis e riconduce i rapporti omosessuali e la pederastia allo stupro; tuttavia non desina dal richiamare la natura squisitamente morale del reato, capace di attirare l'ira divina. L'applicazione della norma sembrerebbe poi fare riferimento ad un provvedimento perduto di Giustiniano, menzionato da Procopio come 'nuova legge' (Proc., Anekd. 11,34.36), che concretizza le "exsquisitae poene subdantur infamae" già previste da una legge di Costanzo e Costante. Pene che Teodosio, Valentiniano ed Arcadio precisano nel pubblico rogo. ${ }^{7}$

La prefata legge di Costanzo e Costante stigmatizza l'omosessualità passiva, con la formula "vir nubit in feminam" e l'icastica metafora del "Venus mutatur in alteram formam". ${ }^{8} \mathrm{Il}$ testo normativo risponde al proliferare delle possibilità di poter contrarre rapporti sessuali con maschi nei luoghi di meretricio; possibilità che a Roma è ben attestata. Tanto che si suole intervenire con l'arresto in flagrante e la pronta asportazione dai bordelli: "occupatos atque omnibus
4. Proc. Caes., De bell. Ghot. 13-20; Hopkins (1963:62-80).

5. Liut., Ant. VI, 4-10.

6. Proc., Anekd. 9, 1-28; Cesaretti (2001).

7. [in modo che i rei d'infamia subiscano le pene prestabilite] Coll. 5, 3, 1-2.

8. [l'uomo che copula qual fosse femmina]; [Venere si trasforma in qualcos'altro] C. Th $9,7,3$. 
9. [dopo averli arrestati, portati tutti fuori dai bordelli maschili] Coll. 5, 3, 1-2.

10. [sia più a lungo macchiata dalla contaminazione dell'effeminatezza maschile, e che quella forza agreste, che discende dai fondatori, mollemente infranta dal popolo, infligga biasimo ai secoli dei nostri fondatori] Coll. 5, 3, 1-2.

11. [che nulla hanno di diverso dalle donne] Coll. 5, 3, 1-2.

12. [coloro che si fanno eunuchi] Just. Nov., 142. eductos virorum lupanaribus". ${ }^{9}$ La norma vuole salvaguardare una strategia di rappresentazione della virilità romana. Non meraviglia la presenza dell'espressione adoperata nel testo: “diutius effeminati in viros pudores contaminatione foedari et agreste illud a priscis conditoribus robur fracta molliter plebe tenuatum convicium saeculis vel conditorum inrogare" ${ }^{10}$ Il legislatore evoca la virilità della Roma originaria, che si esprime nella pienezza della forza agreste dei primi romani. Una capacità che è prima di tutto fecondativa, quale garanzia di successo soprattutto in campo militare.

Si osserva come la produzione legislativa si muova dalla condanna del comportamento di coloro che "nihilque discretum habere cum feminis occupatos" ad una censura totale del fenomeno. ${ }^{11}$ Con Giustiniano ad essere aborrito non è dunque più il 'farsi donna' tradendo la propria virilità, ma il farsi qualcos'altro: il comportarsi come eunuco, quale uomo 'incompleto'. Tant'è che compare l'espressione: "Qui eunucos faciunt". ${ }^{12} \mathrm{Si}$ chiude il cerchio. Ormai eunuchia e passività sono un sinolo imprescindibile anche nella letteratura normativa. Un incunabolo che giustifica la 'reificazione' rivendicata nel più lungo periodo da Andrea di Salos.

La limitata capacità sessuale degli eunuchi ha davvero ad incidere l'inconscio collettivo e la condizione di uomo 'incompleto' viene sentita effettivamente come minus quam. E se qualsiasi maschio romano e cristiano è ritenuto dalla legge un potenziale imperatore, l'eunuco viene escluso per varie ragioni. L'imperatore è anzi tutto immagine di Dio e per essere tale deve presentarsi completo nella sua fisicità. Ogni menomazione è ostativa all'accesso al regno (Leo III, Ekloga 740). Si deve evitare di restituire un'icona deformata della divinità. Il 'santo' imperatore è pure incarnazione della fecondità della natura. Egli rappresenta la forza generativa, come dimostra il perduto affresco del bagno di Leone VI, con una dama raffigurata sulle ginocchia imperiali a mimare un atto sessuale (Magdalino, 1988:97-118). L'incapacità generativa diviene così causa ostativa per l'accesso al trono.

Un atteggiamento culturale che si addolcisce sotto Leone VI, che con due novelle si limita a vietare il matrimonio degli eunuchi, perché non può prevedere la procreazione, il fine ultimo dell'istituto (Leo VI, Nov. 98). Un criterio che non risponde solo a ragioni pratiche, ma può essere considerato un sottoprodotto, che costituisce un retaggio di quelle elaborazioni la cui vitalità si spalma nel più lungo periodo.

Al contempo il basileus permette agli eunuchi di adottare figli, per impedire la dispersione di importanti risorse che questi, lavorando a corte o nelle case patrizie, riescono ad accumulare (Leo VI, Nov. 38). Leggi che sono un'appendice della temperie culturale in evoluzione, che arriva a minimizzare la castrazione, fino a divenire pratica seguita pure da alcuni dei membri delle famiglie nobili più in vista, che attraverso $i$ congiunti eunuchi incardinati a corte mantengono posizioni d'autorità nonostante i frequenti cambi di regime.

È pure noto il caso di Bagoas, che nel sec. XI arriva persino a castrarsi per conservare col proprio aspetto efebico le attenzioni maschili, quali quelle dei colleghi di status, ma fors'anche di gente più anziana. Al di là delle velleità dei singoli beneficiari delle azioni del welfare imperiale, sorge il dubbio che questo orfanotrofio fosse un luogo di approvvigionamento di eunuchi. Qui forse si crea una particolare condizione di fatto, che permette la selezione di soggetti avvezzi a raggiungere questa condizione (Miller, 2003:316).

Un'incapacità a generare come antitesi della mascolinità, stigmatizzata da un aneddoto di Liutprando, che però non esita a fare dono di eunuchi a Costantino 
VII durante la sua prima visita costantinopolitana (Liut., Ant. IV 9). Questi racconta che il margravio di Spoleto, Teobaldo, viene fermato nel proposito d'operare l'eunuchizzazione di un prigioniero bizantino dalla di lui moglie. Colpiscono le accorate parole della lamentatio muliebre, che perorano la causa dell'uomo condannato all'evirazione:

quale guerra, di grazia, più crudele potete portare alle donne, o che cosa di più grave potete portar via ad esse, del fatto che andiate a gara a tagliare $\mathrm{i}$ testicoli agli uomini loro, nei quali c'è il ristoro del nostro corpo e, cosa più importante di tutte, v'è la speranza della prole nascitura? Mentre li castrate, togliete non ciò che è loro ... (Liut., Ant. V, 32; Cantarella, 2004:49)

Eppure è la sottile ironia che si instaura nel botta e risposta fra la donna ed il margravio, a spingere a desistere dal proposito. Allorché quest'ultimo chiede incuriosito: "cosa potrò togliere a tuo marito se si ribella di nuovo?"; la donna incalza col motteggio: "occhi (...), narici, mani e piedi (...), lascia quel che è mio". Si raffronta un malcelato disgusto, proprio del narratore occidentale, verso la categoria, che suscita non l'empatia verso la donna, ma piuttosto il riso.

Per converso il lamento dimostra come siano proprio le donne a dolersi delle conseguenze della presenza di questa categoria nella vita quotidiana. Gli eunuchi sono inutili in una società che organizza la vita femminile in funzione del 'mercato' matrimoniale. L'aumento degli eunuchi fa venire meno una preziosa risorsa maschile per la domanda matrimoniale.

La loro esistenza attenta pure alla procreazione tout court. La castrazione scema non solo la possibilità di usufruire dei piaceri sessuali, ma le menoma della loro funzione generatrice, rendendo impossibile la concezione della prole. L'esistenza degli eunuchi si oppone allora anche alla condizione della femina byzantina.

\section{I tentativi di apologia di genere: 1'opera di Teofilatto di Ocrida ed un episodio della vita di S. Metrios}

Quello che progressivamente si va definendo come genere autonomo, appare vittima di un pregiudizio culturale legato all'esercizio della propria sessualità secondo modalità 'non convenzionali' ed, indi, alla vessata possibilità di esprimere la propria virilità. Si comprende bene come da un certo punto in poi si sia sentita l'esigenza di un'apologia di genere. Teofilatto di Ocrida (1050 -1109 d.C.) allora pone mano alla stesura di un pamphlet, constante in un'opera che è diretta al proprio fratello: l'eunuco Demetrio. Un trattatello che sembra destinato ad un'ampia circolazione, in cui il vescovo bulgaro cerca un punto di vista alternativo per interpretare la condizione di eunuco e, al contempo, risponde adeguatamente a tutti i loci pregiudizievoli, quali i luoghi comuni e quelli della retorica, che si sono consolidati attorno al tema. Una scelta, quella di difendere l'eunuchia, che non può essere estranea ad interessi familiari. La preoccupazione dell'apologeta emerge chiaramente nel proemio in metro giambico, laddove s'afferma:

Non si deve condannare in blocco gli eunuchi che sono virtuosi, per il fatto che se ne veda uno malvagio. Non l'eunuchismo è semplicemente causa di malvagità, ma la libera volontà, che generando talvolta il male, sia negli uomini interi che nei castrati, tuttavia in questi lo produce minore e meno forte (Mercati, 1925:370-372). 
Partendo da questo assioma Teofilatto può intessere la lode della continenza degli eunuchi bizantini, che spesso diventano monaci e persino vescovi. Li addita pure come uomini dediti sovente alla castità e alla pratica delle buone opere, quale dato che giustifica la fama di santità di alcuni di loro. Costoro devono essere giudicati non per la loro diversità, ma come un qualsiasi soggetto, che ha la possibilità di conquistare le vette spirituali, perché può essere casto e virtuoso, ne più, né meno di un qualsiasi altro uomo 'completo'. Il trattatello arriva persino a postulare che la castrazione sia il miglior metodo per conservare la castità dei più giovani.

Un eccesso forse, se non una vera e propria novità, che rileva sul piano diacronico. Si propone una soluzione sui generis rispetto alla tradizione della stessa Chiesa, che non ha mai assolto Origene (185-254 d.C.), il quale ha optato per l'auto-evirazione al fine di mantenersi casto. Un gesto che ha anche ipotecato la propria consacrazione sacerdotale, quale forzatura delle norme canoniche; cosa che lo ha reso reo di eresia. Una soluzione estrema, che in ultima analisi deve essere liquidata piuttosto come mero espediente retorico, quale espressione polemica posta a rafforzamento dell'apologia. Con Teofilatto siamo di fronte ad una forzatura delle strutture del pensiero, non certo ad un punto di rottura rispetto ad una serie di elaborazioni che persistono nel tempo.

È noto che il Concilio di Nicea I del 325 d.C., corregge un'interpretazione diffusa di un passo di Matteo: "Vi sono infatti eunuchi che sono nati così dal ventre della madre; ve ne sono alcuni che sono stati resi eunuchi dagli uomini, e vi sono altri che si sono fatti eunuchi per il regno dei cieli. Chi può capire, capisca" (Mt 19,12).

I 318 padri condannano l'auto-castrazione posta in essere in alcuni ambienti ereticali, che la praticano ai fini di contenere la libido dei propri adepti e non solo. Alla mutilazione rituale i padri oppongono la pratica dell'ascesi, quale base spirituale per l'esercizio della castità e della continenza della carne. L'eunuchia poi costituisce causa ostativa in Occidente per l'accesso ai ranghi del sacerdozio, specie all'episcopato, quale elaborazione peculiare, rispetto a quel che avviene a Bisanzio (Höfert; Mesley; Tolino, 2017:43-65; 192-211; Tracy, 2013).

Eppure per poter avvallare il raggiungimento della condizione di eunuco si ricorre ad espedienti dal carattere affabulatorio, ciò a dimostrazione della vitalità delle elaborazioni concernenti il tema. Per giustificare lo status interviene persino l'adynaton, come nel caso della vita di S. Metrios, che castra il figlio e lo rende eunuco dopo una visione angelica (Herrin, 1993:216). Una pratica, giustificata solo dall'estrema indigenza di chi la subisce, viene riammessa attraverso una deroga straordinaria effettuata a mezzo del meraviglioso. Una deroga che non è applicabile tout court e costituisce un caso eccezionale. Difatti non permette in alcun modo la cristallizzazione di un precedente valido ad avvallare la prassi. La visione angelica è solo un espediente a margine del fenomeno. Va letta nel quadro totalizzante di un racconto come quello agiografico, che non è solo edificante, ma il più delle volte anche apologetico. L'evocazione del meraviglioso implica l'esistenza di dubbi circa la liceità della condotta, che deve essere giustificata con ogni mezzo, forse perché incide in negativo la fama, mettendo in discussione il carisma di santità.

Le elaborazioni concernenti la sessualità ed il suo esercizio da parte del maschio esigono opportune giustificazioni, che vengono fornite in diverso modo, allorché viene impedito. Queste costituiscono a loro volta delle elaborazioni, che rilevano sul piano sincronico e diacronico, rispetto alla reale percezione del fenomeno da parte dell'uomo bizantino. 


\section{Conclusioni}

La libido degli eunuchi, espressa nonostante gli impedimenti più o meno rilevanti dovuti alla menomazione subita, è un problema che si inserisce nel normale menage della vita sessuale dell'uomo bizantino e può suscitare fantasie specie nel maschio, offrendogli occasioni di trasgressione. Si va così a costituire un'alternativa possibile per questi ultimi nel vivere questa componente della vita.

Una sessualità quella degli eunuchi che, al contempo, funge da 'catalizzatore' nel processo di costruzione di un tertium genus, che prende sommessamente corpo nel più lungo periodo oltre ogni pregiudizio; anzi costituisce la risposta ed il contrappeso opposto all'opinione comune. Una sessualità depotenziata che pone alcune problematiche, specie allorché si deve spiegare ogni comportamento che eccede lo stato di uomo 'incompleto'. Le azioni prettamente virili ed il valore militare, che non rientrano nello stereotipo di un soggetto che si vuole necessariamente effeminato, aprono ad un vero e proprio 'dilemma retorico' (Herrin, 1993:218; Neil; Garland, 2016). Ammettendo la propensione alla virtù guerriera, si mette in discussione il codice di auto-rappresentazione della virilità del maschio bizantino e ciò implica pure la percezione del suo opposto: la femina. Viene così investito l'intero universo di rappresentazione delle categorie maschio/femmina del periodo di riferimento. L'essere "né uomini, né donne" fa degli eunuchi gli attori di un "dramma" etero-referenziale (Ducellier, 1980:21). La loro libido poi stimola condotte sessuali alternative, che si oppongono all'istituto del matrimonio e alla sua immediata finalità: la procreazione, costituendo un pericolo non solo sul piano della morale. Pratiche che vanno perciò condannate dalla religione. Voluttà che, in ultima analisi, hanno a ridisegnare la percezione della mascolinità del periodo di riferimento e vanno sia combattute a suon di prediche, sia cesurate dalle norme. Oppure sottaciute per salvare l'idea di virilità, almeno così come si è cristallizzata. 


\section{Q Bibliografia}

» Bekker, J. (ed.) (1838). Theophane Continuatus, Chronografia. Bonn: Weber.

" Cantarella, E. (2006). Secondo natura. La bisessualità nel mondo antico. Roma: Feltrinelli.

»Cantarella, G.M. (2004). Una sera dell'Anno Mille. Milano: Garzanti.

"Carile, R. A. (2003a). "La prossemica del potere: spazi e distanze nei cerimoniali di corte". En: Uomo e spazio nell'Alto Medioevo. Atti della L Settimana di Studio del Centro Italiano di Studi sull'Alto Medioevo (4-8 aprile 2002, Spoleto). Spoleto: Centro Italiano di Studi sull'Alto Medioevo, 589-656.

»Carile, R. A. (2003b). “Creduntaliud romana palatia caelum. Die Ideologie des Palatium in Konstantinopel dem Neuen Rom". En: König, M.; Bolognesi Recchi Franceschini, E.; Riemer, E., (eds.), Palatia. Kaiserpaläste in Konstantinopel, Ravenna und Trier. Trier: Rheinisches Landesmuseum, 27-32.

»Cesaretti, P. (2001). Teodora. Ascesa di una imperatrice. Milano: Mondadori.

" Comparetti, D. (1895). Procopio di Cesarea, La Guerra Gotica. Roma: Forzani.

" Corsi, P. (1991). "L'eunco". En: Condizione umana e ruoli sociali nel Mezzogiorno normanno-svevo: Atti delle none Giornate normanno-sveve (17-20 ottobre 1989, Bari). Bari: Dedalo, 251-277.

»Dagron, G. (2007). "From the mappa to the akakia: symbolic drift". En: Hagit, A., ter Haar Romeny, R. B. (eds.), From Rome to Constantinople: Studies in Honor of Averil Cameron. Leuven-Paris: Peteers, 78-110.

»Dalla, D. (1987). Ubi Venus mutatur, Omosessualità e diritto nel mondo romano. Milano: A. Giuffrè.

»Ducellier, A. (1980). Il dramma di Bisanzio. Napoli: Liguori.

» Guillard, R. (1943). "Les eunuques dans l'empire byzantin. Étude de titulature et de prosopographie byzantins", Revue des Études byzantines 1, 196-238.

" James, E. (1997). Women, Men, and Eunuchs: Gender in Byzantium. Front Cover. Abingdon: Taylor \& Francis Group.

" James, E. (2009). "Men, woman, eunuch: gender, sex and power". En: Haldon, J. (ed.) (2009), A Social History of Byzantium. Chichester: Wiley Blackwell.

» Každan, A.P. (1982). People and power in Byzantium: an introduction to modern Byzantine studies. Washington: Dumbarton Oaks.

» Kramers, J. H. (ed.) (1964). Ibn Ḥawqal, Configuration de la Terre (Kitāb Șūra al-Ard). Paris: Maisonneuve et Larose, vol. I, 198-199.

" Hadley, D. (1998). Masculinity in medieval Europe. Harlow: Longman.

» Haldon, J. (2009). A Social History of Byzantium. Chichester: Wiley Blackwell.

» Herrin, J. (2008). “Eunuchi”. En: Bisanzio. Milano: Corbaccio, 207-218.

" Höfert, A.; Mesley, M.; Tolino, S. (2017). Celibate and Childless Men Placed into a Shared Focus: Ruling Eunuchs and Bishops Between the Intersections of Power. Abigndon: Routledge.

» Hopkins, K. (1963). "Eunuchs in Politics in the Later Roman Empire", PCPhS 9, 62-80. 
" Magdalino, G.C.P. (1988). "The Bath of Leo the Wise and the Macedonian Renaissance Revisited: Topography, Iconography, Ceremonial, Ideology", DOP 42, 97-118.

" Mango, C. (1982). "The life of St Andrew the fool reconsidered", Rivista di Studi Bizantini e Slavi2, 297-313.

" Mercati, S. G. (1925). "Poesie di Teofilatto di Bulgaria”, Studi Bizantini 1, 348-372.

» Miller, T.S. (2003). The Orphans of Byzantium, Child Welfare in the Christian Empire. Washington: The Catholic University of American Press.

» Neil, B.; Garland, L. (2016). Questions of Gender in Byzantine Society. Abigndon: Routlege.

»Odorico, P. (ed.) (1995). Digenis Akritas, Poema anonimo bizantino. Firenze: Giunti.

» Pontieri, E. (ed.) (1925-1928). Gaufredo Malaterra, De rebus gestis Rogerii Calabriae et Siciliae Comitis et Roberti Guiscardi Ducis fratris eius, RIS V/1. Bologna: Zanichelli

" Patlagean, E. (1992). Santità e potere a Bisanzio. Milano: Il Saggiatore.

» Ravegnani, G. (1989). La corte di Giustiniano. Venezia: Jouvence.

"Ringrose, K. M. (2007). The Perfect Servant: Eunuchs and the Social Construction of Gender in Byzantium. Chicago-London: The University of Chicago Press.

» Rydén, L. (ed.) (1995). The Life of St Andrew the Fool. Uppsala: Acta Universitatis Upsaliensis.

"Spadaro, M. D. (2006). "Cli eunuchi nell'impero bizantino". En: Comportamenti e immaginario della sessualità nell'Alto Medioevo. Spoleto: Centro italiano di studi sull'Alto Medioevo, 535-566.

"Thomas, J.; Costantinides Hero, A. (eds.) (2000). Byzantine Monastic Foundation Documents. Washington: Dumbarton Oaks.

» Toucher, S. (2002). Eunuchus in Antiquity and Beyond. London: Classical Press of Wales and Duckworth.

"Tougher, S. F. (1997). "Byzantine Eunuchs: An Overview, with Special Reference to Their Creation and Origin". En: James, L. (ed.) (1997), Men, woman, eunuch: Gender In Byzantium. London: Routledge, 168-184.

» Tracy, L. (2013). Castration and Culture in the Middle Ages. Cambridge: Brewer. 
